Supporting Information for

\title{
Profiling Glucose-Stimulated and M3 Receptor-Activated Insulin Secretion Dynamics from Islets of Langerhans Using an Extended-Lifetime Fluorescence Dye
}

\author{
Joel E. Adablah, Yao Wang, Matthew Donohue, and Michael G. Roper* \\ Department of Chemistry and Biochemistry, Florida State University, \\ 95 Chieftain Way, Tallahassee, FL 32306
}

\begin{abstract}
Additional details illustrating the optical setup and pressure-driven perfusion system. Mathematical derivations for immunoassay equilibrium equations are provided, as well as a table containing the properties of SeTau-647 and Cy5. Supplementary glucose-stimulated insulin secretion profiles for single and grouped murine in addition to grouped human islets are also shown. Control experiments are presented to demonstrate insulin secretion was unsynchronized without carbachol (CCh).
\end{abstract}




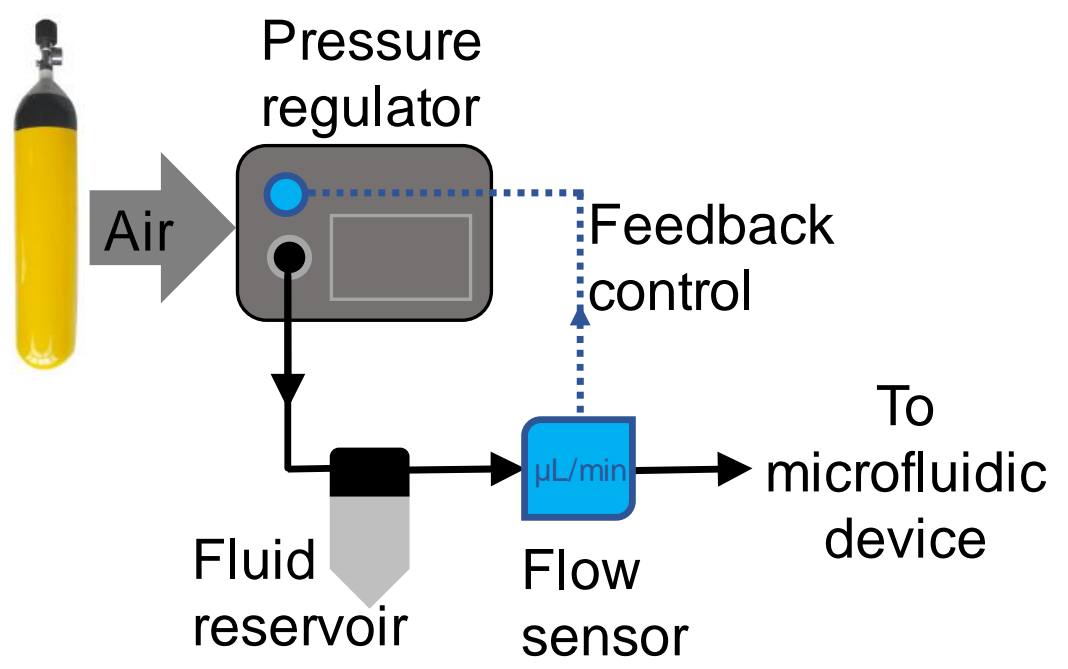

Figure S1. Active pressure-driven perfusion system. A piezoelectric pressure regulator was used to drive reagents from fluid reservoirs through an inline flow sensor to the microfluidic device. Flow rates were monitored by the flow sensor which enabled online variations in the pressure to the fluid reservoirs via a feedback control loop. 


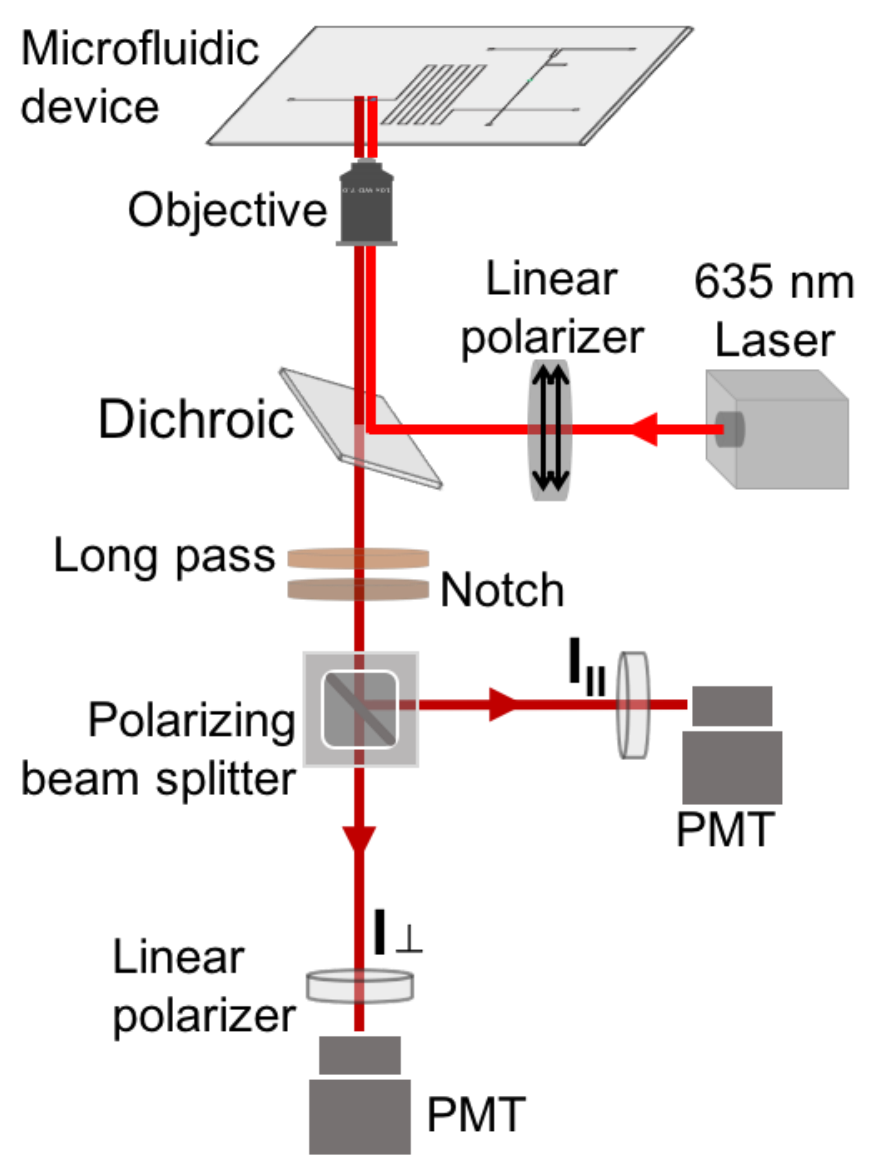

Figure S2. Schematic of optical setup. Light from a $635 \mathrm{~nm}$ laser was passed through a neutral density filter, into a multimode fiber optic bundle, collimated, and then depolarized (not shown on the figure). This depolarized light was passed through a linear polarizer, reflected by a $660 \mathrm{~nm}$ dichroic mirror, and focused into the microfluidic channel using a 40X, 0.6 NA microscope objective. Laser-induced fluorescence was collected by the objective, transmitted through the dichroic, a $665 \mathrm{~nm}$ long pass filter, and a 635 notch filter. The parallel and perpendicular components of laser-induced fluorescence passed though complementary linear polarizers and were measured by individual photomultiplier tubes (PMT). 


\section{Derivation for calculated B/F vs. [Ins] calibration curve}

In an insulin competitive immunoassay, the reaction takes place as follows:

$$
\operatorname{lns} s^{*}+\operatorname{Ins}+A b \rightleftharpoons A b-\operatorname{lns}{ }^{*}+A b-\ln s
$$

The dissociation constants of the antibody-insulin complex and the antibody-insulin* complex are defined as:

$$
\begin{aligned}
K_{d} & =\frac{[A b][\operatorname{Ins}]}{[A b-\operatorname{lns}]} \\
K_{d}^{*} & =\frac{[A b]\left[\operatorname{lns} s^{*}\right]}{\left[A b-\operatorname{lns} s^{*}\right]}
\end{aligned}
$$

Where, $\mathrm{K}_{d}$ is the dissociation constant of antibody-insulin complex, and [Ab], [Ins], and [Ab-Ins] are the equilibrium concentration of antibody, insulin, and antibody-insulin complex, respectively. Similarly, $\mathrm{K}_{d}{ }^{*}$ is the dissociation constant of the antibody-insulin* complex, and $\left[\operatorname{lns}^{*}\right]$ and $\left[\mathrm{Ab}-\operatorname{Ins}^{*}\right]$ are the equilibrium concentration of insulin* and antibody-insulin ${ }^{*}$ complex, respectively.

Based on conservation of mass, equations for the initial concentrations of insulin* (Inso*), insulin (Inso), and antibody ( $\left.A b_{0}\right)$ can be written (equations 3,4 , and 5 , respectively):

$$
\begin{gathered}
{\left[\operatorname{Ins} 0^{*}\right]=\left[\operatorname{Ins}{ }^{\star}\right]+\left[\text { Ab-Ins }{ }^{*}\right]} \\
{[\operatorname{Ins} 0]=[\operatorname{Ins}]+[\text { Ab-Ins }]} \\
{\left[A b_{0}\right]=[A b-\operatorname{lns}]+\left[\text { Ab-Ins }{ }^{\star}\right]+[A b]}
\end{gathered}
$$

Given initial concentrations of reagents and dissociation constants, the measured ratio of bound-to-free Ins $^{*}(B / F)$ is calculated from equations 1-5 using: 


$$
\frac{\mathrm{B}}{\mathrm{F}}=\frac{\left[\mathrm{Ab}-\operatorname{Ins} \mathrm{s}^{*}\right]}{\left[\operatorname{lns} \mathrm{s}^{*}\right]}
$$

For calculations of the $\mathrm{B} / \mathrm{F}$ ratios for Figure $2 \mathrm{~A}, \mathrm{Kd}_{\mathrm{d}}$ and $\mathrm{Kd}^{*}$ were set to $10 \mathrm{nM}$ each, and $\left[\operatorname{lns}^{*}{ }_{0}\right]$ and $\left[\mathrm{Ab}_{0}\right]$ were each set to $24 \mathrm{nM}$. The B/F ratios for each concentration of Inso in the calibration curve were then calculated, followed by the anisotropy of the resulting mixture using values in Table $\mathrm{S} 1$ and equations 1-3 given in the text. 
Table S1. Summary of molecular weights and optical properties of all fluorescently labeled immunoassay products

\begin{tabular}{|c|c|c|c|c|}
\hline $\begin{array}{l}\text { Labeled } \\
\text { product }\end{array}$ & MW (kDa) & $\boldsymbol{\theta}(\mathbf{n s})^{a}$ & $\tau(\mathrm{ns})$ & $r_{0}$ \\
\hline $\operatorname{lns}{ }_{\text {Cy5 }}^{*}$ & 6.6 & 1.30 & 1.0 & $0.37^{b}$ \\
\hline $\operatorname{lns}{ }^{*}{ }_{\mathrm{Cy} 5}-\mathrm{Ab}$ & 156.6 & 31.45 & 1.0 & $0.37^{b}$ \\
\hline Ins $^{*}{ }_{\text {SeTau- } 647}$ & 7.6 & 1.50 & 3.2 & $0.40^{c}$ \\
\hline $\operatorname{lns}_{\text {SeTau-647 }}^{*}-\mathrm{Ab}$ & 157.6 & 31.65 & 3.2 & $0.40^{c}$ \\
\hline
\end{tabular}

${ }^{a}$ : Values were computed using reference 32 in the text and the MW for each respective species.

${ }^{b \& c}$ : Values were obtained from references 43 and 44 in the text, respectively. 

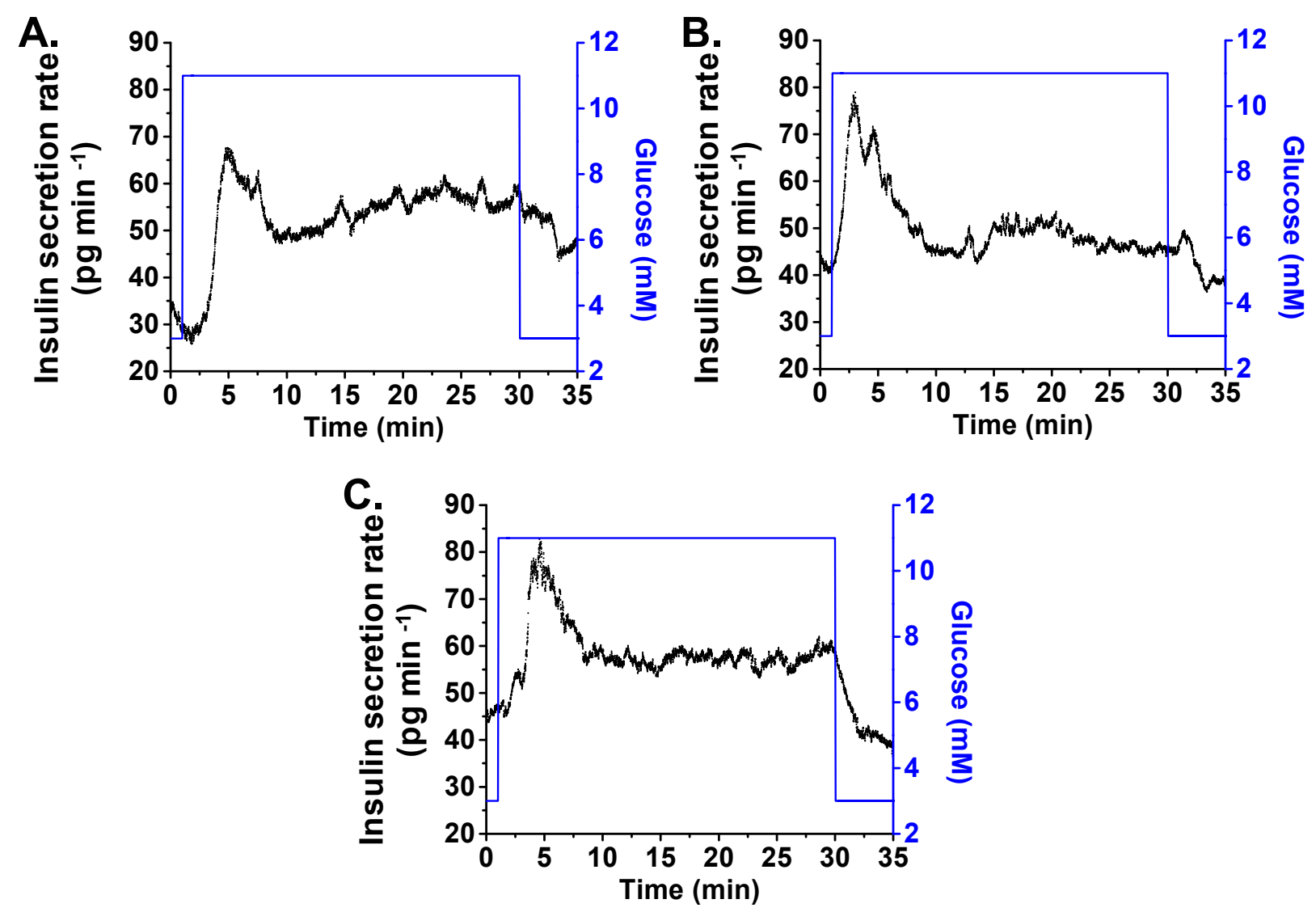

Figure S3. GSIS profile from 3 single murine islet experiments. A single murine islet in each experiment was stimulated with $11 \mathrm{mM}$ glucose for 30 min during which insulin release was measured. In each plot, the black trace (left $y$-axis) is the rate of insulin secretion from the islet. The blue profile (right y-axis) represents the glucose challenge. 

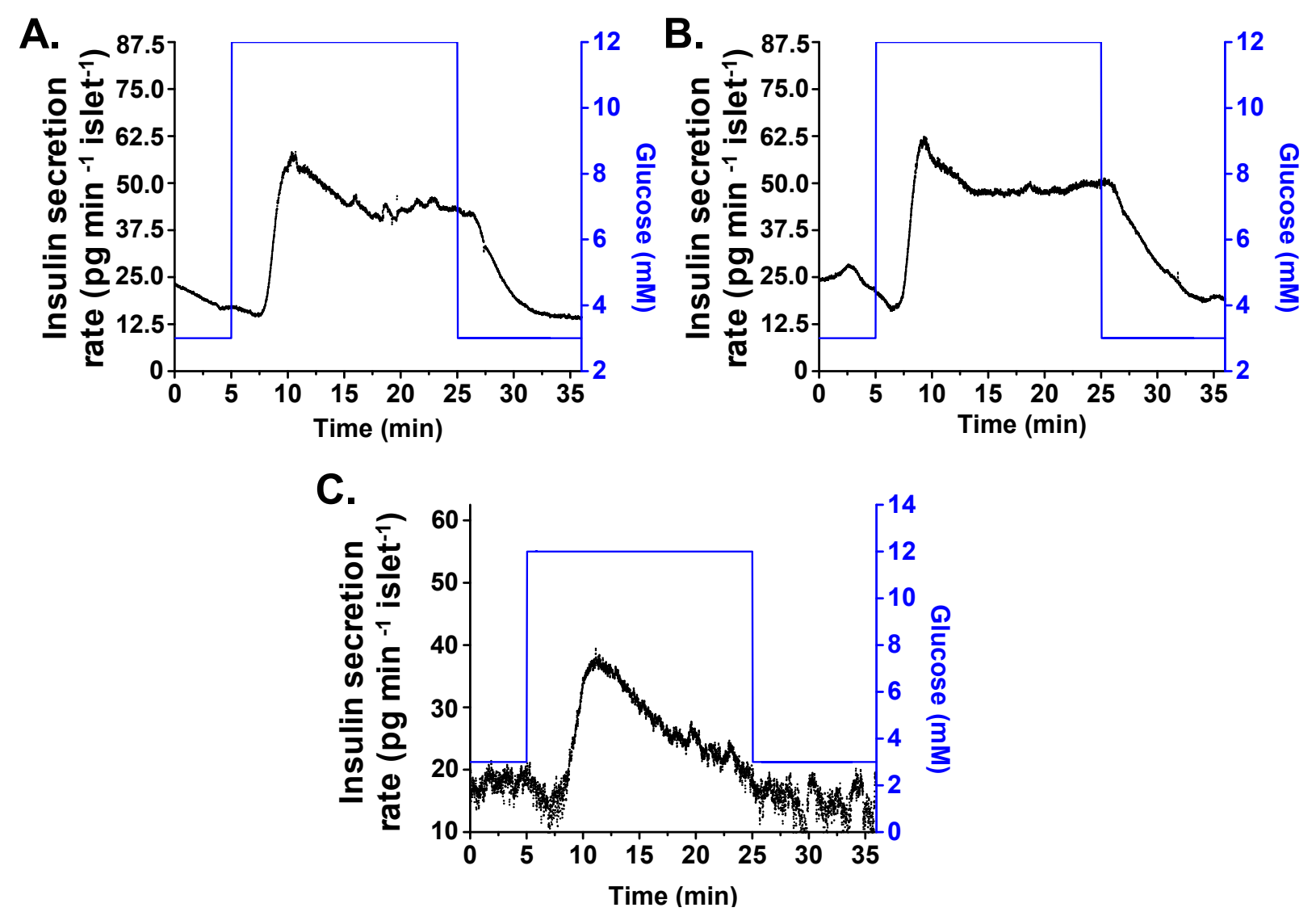

Figure S4. GSIS from 3 grouped murine islet experiments. Insulin release rates are profiled with the black trace (left axis). Each experiment consisted of 4 islets in the chamber. Islets in each experiment were stimulated with $12 \mathrm{mM}$ glucose for $20 \mathrm{~min}$ (blue profile, right axis) during which insulin release was measured. 


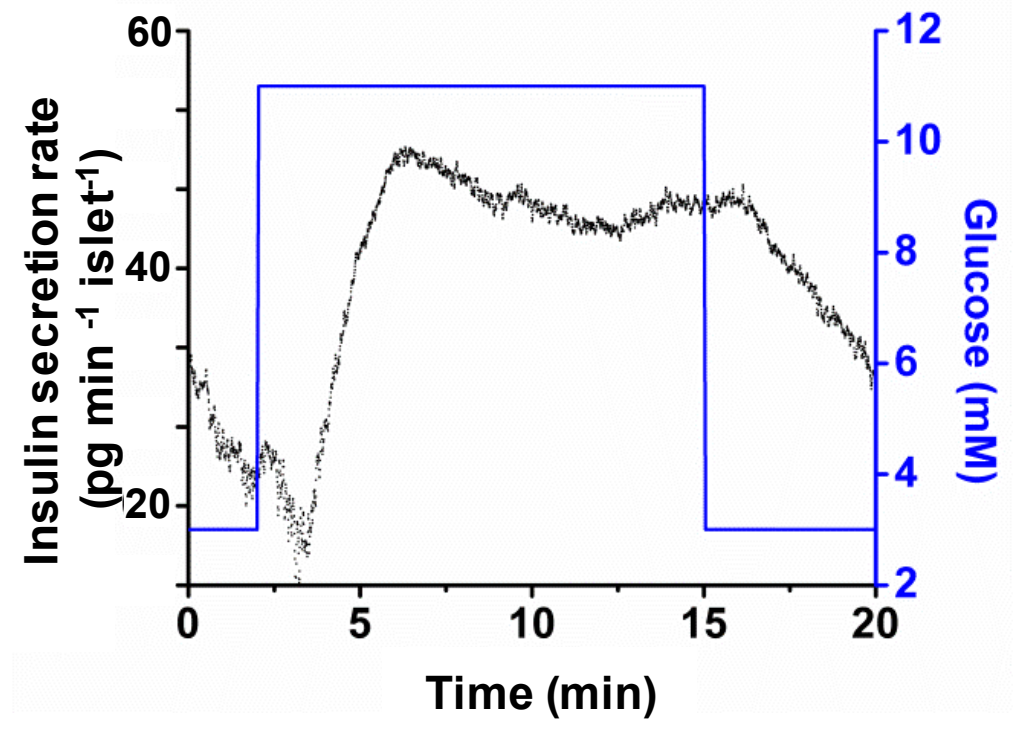

Figure S5. GSIS from a group of 3 healthy human islets. Insulin secretion (black trace, left $y$-axis) was measured from the 3 islets during a 13 min delivery of $11 \mathrm{mM}$ glucose (blue trace, right y-axis). 

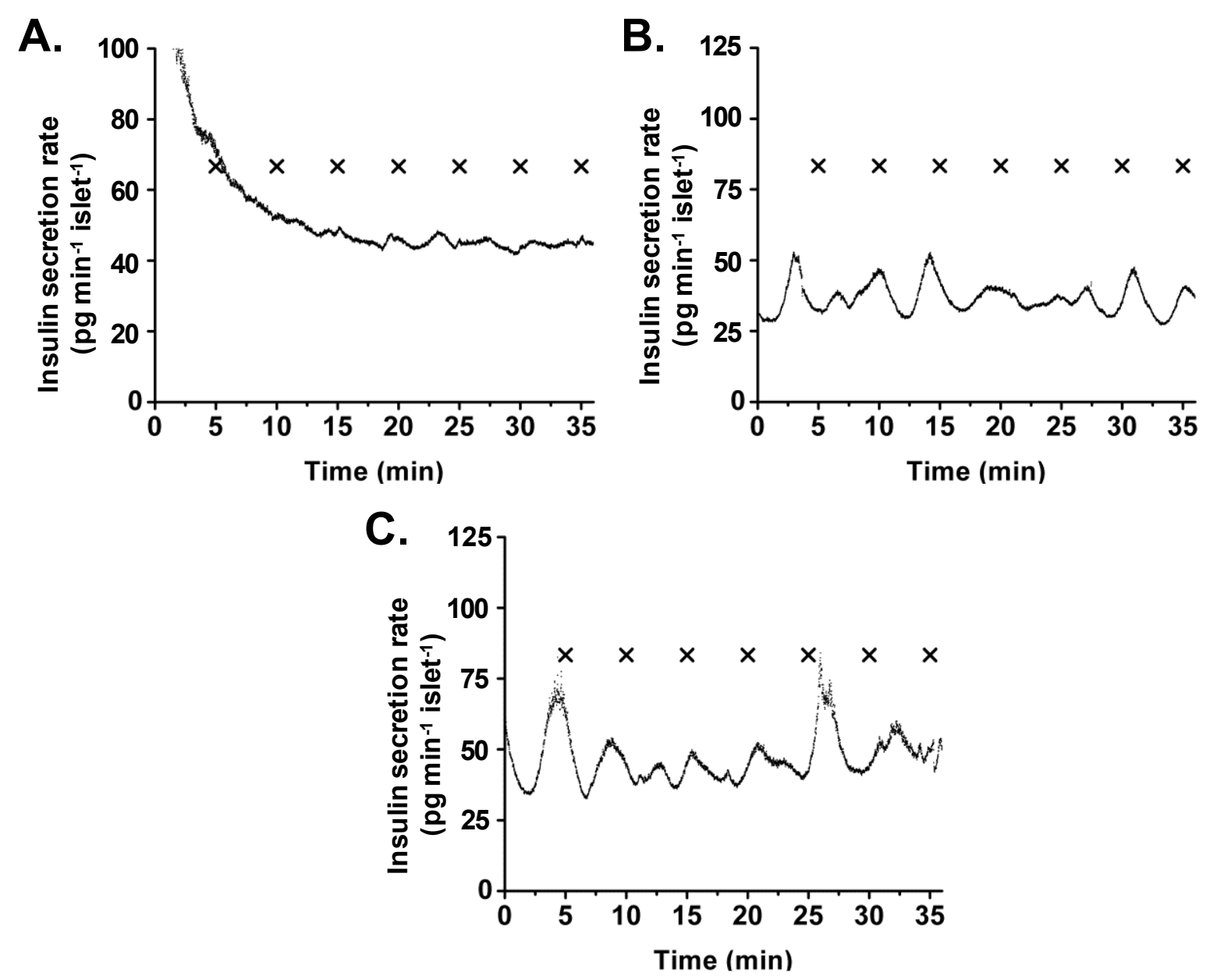

Figure S6. Control pulses without CCh. Three control experiments were performed with murine islets exposed to constant $11 \mathrm{mM}$ glucose. Five murine islets were used in $\mathbf{A}$ and 4 in $\mathbf{B}$ and $\mathbf{C}$. The ' $x$ ' in each plot indicates the time the fluidic system switched. The tail end of the phase 1 burst is visible in the early stages of insulin measurement in $\mathbf{A}$. Oscillations observed in $\mathbf{B}$ and $\mathbf{C}$ are short-lived and irregular, and showed no dependence to the glucose pulses applied. 